\title{
Gray Matter Atrophy And Corresponding Impairments In Connectivity In Patients With Anti-N- Methyl-D-Aspartate Receptor Encephalitis
}

\section{Yuanyuan Guo}

The First Affiliated Hospital of Anhui Medical University https://orcid.org/0000-0002-1295-7411

\section{Xinyi LV}

The First Affiliated Hospital of USTC

\section{Juanjuan Zhang}

The First Affiliated Hospital of Anhui Medical University

\section{Chenglong Li}

The First Affiliated Hospital of Anhui Medical University

\section{Ling Wei}

The First Affiliated Hospital of Anhui Medical University

\section{Nong Zhou}

The First Affiliated Hospital of Anhui Medical University

\section{Jinping Xu}

Shenzhen Institutes of Advanced Technology

Yanghua Tian ( $\nabla$ ayfytyh@126.com )

The First Affiliated Hospital of Anhui Medical University

\section{Kai Wang}

The First Affiliated Hospital of Anhui Medical University

\section{Research Article}

Keywords: Anti-N-methyl-D-aspartate receptor encephalitis, Cognitive impairment, Functional connectivity, Voxel-based morphometry, Gray matter volume.

Posted Date: January 3rd, 2022

DOI: https://doi.org/10.21203/rs.3.rs-1191805/v1

License: (c) (1) This work is licensed under a Creative Commons Attribution 4.0 International License. Read Full License 


\section{Abstract}

Anti-N-methyl-D-aspartate receptor (NMDAR) encephalitis is a severe autoimmune disease that is commonly accompanied by cognitive impairment and various neurological and psychiatric symptoms, advanced image analyses help explore the pathogenesis of this disease. Therefore, this study aimed to explore specific structural and functional alterations and their relationship with the clinical symptoms of anti-NMDAR encephalitis. In this study, twenty-two patients with anti-NMDAR encephalitis after the acute stage and 29 controls received cognitive assessments and magnetic resonance imaging. Grey matter atrophy was measured using voxel-based morphometry, and functional alterations in abnormal regions were subsequently investigated using resting state functional connectivity (RSFC). Finally, correlation analyses were performed to explore the associations between imaging alterations and cognitive assessments. The patients demonstrated significant gray matter atrophy in the bilateral triangle part of the inferior frontal gyrus (trilFG.L and trilFG.R) and right precuneus, decreased RSFC between trilFG.L and bilateral Heschl gyrus (HES), decreased RSFC between trilFG.R and HES.R, decreased RSFC between right precuneus and left cerebellum, and increased RSFC between trilFG.R and left superior frontal gyrus. Further correlation analyses showed that the gray matter volume in trilFG.R and decreased RSFC between trilFG.L and HES.R were associated with decreased memory scores, whereas decreased RSFC between trilFG.R and HES.R was marginally correlated with the disease course in patients. In conclusion, this study suggests that cognitive impairments in patients with anti-NMDAR encephalitis may be mainly associated with gray matter atrophy and abnormal RSFC in the trilFG. These findings provide new insights into antiNMDAR encephalitis pathogenesis and help explore potential treatments.

\section{Introduction}

Anti-N-methyl-D-aspartate receptor (NMDAR) encephalitis results from the production of anti-NMDAR GluN1-subunit autoantibodies. This disease can induce numerous neuropsychiatric symptoms, including psychosis, epileptic seizures, dyskinesias, cognitive deficits, consciousness disorders, autonomic instability, and central hypoventilation (Carsten et al., 2016; Dalmau, 2016; Dalmau et al., 2008; Irani et al., 2010; Viaccoz A et al., 2014). NMDAR is widely expressed in the brain and it is highly concentrated in the frontal lobe, hippocampus, and cerebellum (Moscato et al., 2014; Skowronska et al., 2019; Wang and Xiao, 2020). Moreover, it plays a prominent role in synaptic transmission and plasticity processes, which are considered the basis of learning and memory (Lau and Zukin, 2007). Anti-NMDAR antibody production is associated with receptor internalization and synaptic plasticity disruption, further causing long-term memory deficits (Bach, 2014). This disease is clinically diagnosed when anti-NMDAR antibodies are detected in the cerebrospinal fluid, though this procedure is slow and expensive. Moreover, the identification rate of acute stage anti-NMDAR encephalitis using clinical routine magnetic resonance imaging (MRI) is between $11 \%$ and $83 \%$ (Bacchi et al., 2018). Repeated lumbar puncture examinations to detect antibodies are invasive, and conventional MRI in the acute and convalescence phases is less specific than cerebrospinal fluid examination. Therefore, a more convenient and sensitive method to help 
us understand the pathogenesis of this disease and assess its prognosis to provide better treatments is warranted.

Several structural imaging studies have been performed in patients with anti-NMDAR encephalitis in recent years. Finke et al. in 2013 demonstrated that there were no significant changes in gray matter morphology in convalescent patients by using voxel-based morphometry (VBM) analysis (Finke et al., 2013). In contrast, in 2016, significant hippocampus atrophy was found in a study comprising a larger sample size than the previous one (Carsten et al., 2016). Additionally, our previous study has

demonstrated decreased cortical alterations in the language and default mode networks and sub-cortical atrophy of the left hippocampus CA1 body in these patients (Xu et al., 2021), indicating the possible presence of progressive structural brain alterations in patients with anti-NMDAR encephalitis.

In addition to structural MRI, resting state functional MRI (rs-fMRI) can quantify spontaneous fluctuations in the blood oxygen level-dependent signal to measure intrinsic neural activity, which is known as resting state functional connectivity (RSFC) (Brier et al., 2016; Fox and Raichle, 2007). Anti-NMDAR encephalitis has been associated with abnormal hippocampal connectivity and several large-scale networks, such as the default mode network (Finke et al., 2013; Peer et al., 2017). Another rs-fMRI study has demonstrated decreased neural activity in the posterior cingulate cortex, precuneus, and cerebellum of 17 patients with anti-NMDAR encephalitis (Cai et al., 2020). Previous studies have demonstrated that these brain regions and networks mostly showed structural changes (Carsten et al., 2016; Xu et al., 2021), suggesting that these alterations may be accompanied by brain functional impairment. However, most studies on antiNMDAR encephalitis patients have independently examined changes either in neuronal activity or structure (Cai et al., 2020; Carsten et al., 2016; Jinping Xu et al., 2021; Peer et al., 2017). Therefore, we combined VBM and RSFC to focus on structural and related functional alterations in patients with antiNMDAR encephalitis and further explore the underlying mechanisms of the long-term cognitive impairment caused by this disease.

\section{Materials And Methods Participants}

Twenty-four patients with anti-NMDAR encephalitis were enrolled after the acute phase in a grade-three general hospital in Hefei, China. All patients fulfilled the diagnostic criteria for anti-NMDAR encephalitis, including typical clinical features and a positive test for immunoglobulin $\mathrm{G}(\mathrm{IgG})$ NMDAR antibodies in the cerebrospinal fluid using a combination of tissue-based assay and cell-based assay (Dalmau et al., 2011). The detailed patient characteristics are provided in Supplemental Table S1. Thirty normal controls (NCs) with matching sex, age, and education were also included. NCs were negative for anti-NMDAR antibodies and did not have any psychiatric or neurological disorders. The global cognitive function was evaluated using the Montreal Cognitive Assessment (MoCA), and short- and long-term memory were assessed using the Auditory Verbal Learning Test (AVLT, Chinese version) (Table 1). All participants 
provided a written consent statement according to the Declaration of Helsinki, and this study was approved by the ethical committee of Anhui Medical University.

Table 1

Demographic information and clinical measurements

\begin{tabular}{|c|c|c|c|c|}
\hline & $\begin{array}{l}\text { NMDA }(n= \\
\text { 22) }\end{array}$ & $N C(n=29)$ & Statistics & $P$ value \\
\hline Age (years) & $\begin{array}{l}30.54 \pm \\
10.79\end{array}$ & $\begin{array}{l}35.79 \pm \\
15.02\end{array}$ & $\begin{array}{l}t= \\
-1.388\end{array}$ & 0.171 \\
\hline Sex (male:female) & $10: 12$ & $12: 17$ & $\begin{array}{l}\chi 2= \\
0.961\end{array}$ & $0.327^{a}$ \\
\hline Education & $\begin{array}{l}10.54 \pm \\
4.62\end{array}$ & $\begin{array}{l}10.90 \pm \\
3.83\end{array}$ & $\begin{array}{l}t= \\
-0.194\end{array}$ & 0.847 \\
\hline FD & $0.11 \pm 0.05$ & $0.09 \pm 0.04$ & $t=1.340$ & 0.285 \\
\hline MoCA & $\begin{array}{l}23.95 \pm \\
5.40\end{array}$ & $\begin{array}{l}28.03 \pm \\
2.12\end{array}$ & $\begin{array}{l}t= \\
-3.350\end{array}$ & $0.002^{*}$ \\
\hline \multicolumn{5}{|l|}{ AVLT } \\
\hline Immediate recall & $7.67 \pm 2.52$ & $9.91 \pm 1.31$ & $\begin{array}{l}t= \\
-4.099\end{array}$ & $<0.001^{*}$ \\
\hline Delayed recall & $7.31 \pm 4.00$ & $\begin{array}{l}10.44 \pm \\
2.26\end{array}$ & $\begin{array}{l}t= \\
-3.537\end{array}$ & $0.001^{*}$ \\
\hline Disease duration (days) & $\begin{array}{l}46.91 \pm \\
19.59\end{array}$ & - & - & - \\
\hline $\begin{array}{l}\text { Time between first symptom and immune } \\
\text { therapy (days) }\end{array}$ & $\begin{array}{l}20.41 \pm \\
11.55\end{array}$ & - & - & - \\
\hline $\begin{array}{l}\text { Time between first symptom and imaging } \\
\text { (months) }\end{array}$ & $\begin{array}{l}15.14 \pm \\
11.68\end{array}$ & - & - & - \\
\hline $\mathrm{mRS}$ at onset & $3.86 \pm 0.94$ & - & - & - \\
\hline mRS at discharge & $2.73 \pm 1.03$ & - & - & - \\
\hline $\mathrm{mRS}$ at imaging & $0.23 \pm 0.69$ & - & - & - \\
\hline \multicolumn{5}{|c|}{ Note: Data are expressed as mean \pm standard deviation; ${ }^{a} \chi 2$ test results; ${ }^{*} p<0.05$} \\
\hline
\end{tabular}

\section{MRI data acquisition}

MRI scans were obtained from a GE 3.0T MR scanner (Discovery 750; GE Healthcare, Buckinghamshire, UK) on the same day with cognitive assessment. T1-weighted images were acquired using a three- 
dimensional brain volume sequence (3D-BRAVO) using the following parameters: repetition time (TR): $8.16 \mathrm{~ms}$; echo time (TE): $3.18 \mathrm{~ms}$; inversion time (TI): $450 \mathrm{~ms}$; flip angle (FA): $12^{\circ}$; matrix size: $256 \times 256$; field of view (FOV): $256 \times 256 \mathrm{~mm}^{2}$; slice thickness: $1 \mathrm{~mm}$; voxel size: $1 \times 1 \times 1 \mathrm{~mm}^{3}$; and number of sagittal slices: 188. rs-fMRI was performed using the following echo-planar imaging sequence parameters: TR: $2400 \mathrm{~ms}$, TE: $30 \mathrm{~ms}$; acquisition time: 521 s; FA: 90; matrix size: $64 \times 64$; FOV: $192 \times 192$ $\mathrm{mm}^{2}$; slice thickness: $3 \mathrm{~mm}$; voxel size: $3 \times 3 \times 3 \mathrm{~mm}^{3}$; and number of slices: 46 .

\section{Image preprocessing}

The T1-weighted images were preprocessed using Data Processing \& Analysis for Brain Imaging (DPABI; http://rfmri.org/dpabi). First, the quality of each image was visually checked; two participants, one patient and one NC, were excluded due to poor image quality. Subsequently, the images were segmented into gray matter, white matter, and cerebrospinal fluid and transformed into a standard Montreal Neurological Institute space. Then, these images were modulated to preserve regional volume information, and finally smoothed utilizing a 6-mm Gaussian kernel full-width at half maximum and used in the analyses.

Rs-fMRI data were also preprocessed using DPABI with the following main steps: (1) removal of the first seven volumes; (2) slice timing; (3) realignment (participants with a head motion exceeding $3 \mathrm{~mm}$ in any direction or $3^{\circ}$ of angular motion were excluded; one patient had this issue); (4) spatial normalization; (5) resampling to a voxel size of $3 \times 3 \times 3 \mathrm{~mm}^{3}$; (6) smoothing using a Gaussian kernel of 6-mm full-width at half maximum; (7) removal of linear and quadratic trends; (8) exclusion of head motion effects, white matter, cerebrospinal fluid, and global signals using the Friston 24-parameter model (Satterthwaite et al., 2013); (9) temporal band pass filtering $(0.01-0.1 \mathrm{~Hz})$; and (10) "scrubbing" two time-points before and one time-point after obtaining poor-quality images, which were defined as a frame displacement $>0.5$ (Power et al., 2012). All participants had fMRI images for more than half of the total time-points, and these data were analyzed.

\section{Voxel-based morphology}

The VBM analysis were performed using Statistical Parametric Mapping software (SPM12; https://www.fil.ion.ucl.ac.uk/spm/software/spm12/). Differences in whole-brain gray matter volume between the patient and NC groups were explored using a two-sample t-test, with age, sex, and education as covariates. Significance levels were determined using Gaussian random field (GRF) corrections with a voxel-level threshold of $p<0.001$ and cluster-level threshold of $p<0.05$. Finally, the mean gray matter volume of abnormal regions was calculated and compared between groups using a general linear model (GLM) controlled for age, sex, and education in SPSS version 19 (IBM SPSS Inc., Chicago, IL, USA).

\section{RSFC}

RSFC was defined as the Pearson correlation coefficient between the mean time series of seed regions and each voxel in the rest of the brain. Subsequently, all correlation coefficients were converted to $z$ 
values using Fisher's z-transformation to normalize the results. Between-group differences were analyzed using a two-sample t-test, with age, sex, education, and frame-wise displacement (FD) as covariates.

Significance levels were determined using GRF corrections with a voxel-level threshold of $p<0.001$ and a cluster-level threshold of $p<0.05$. Finally, the mean RSFC of abnormal regions was calculated and compared between groups using GLM corrections, controlling for age, sex, education, and FD, in SPSS version 19 (IBM SPSS Inc., Chicago, IL, USA).

\section{Correlation analyses}

Partial correlation analyses were performed between neuroimaging indices, including gray matter volume and RSFC, and clinical measurements, including disease duration, MoCA, and AVLT scores. Age, sex, and education were controlled for gray matter volume, whereas FD was also controlled for RSFC. With limited samples, we provided significance levels of $0.05<p<0.1$ to show trends of significance, and a $p$-value $<$ 0.05 was considered statistically significant.

\section{Results}

\section{Demographic information and clinical measurements}

Twenty-two patients with anti-NMDAR encephalitis (10 males, 12 females) and 29 NCs (12 males, 17 females) were included in the study. There were no significant differences in sex $(\chi 2=0.961$ and $p=$ $0.327)$, age $(t=-1.388$ and $p=0.171)$, education $(t=-0.194$ and $p=0.847)$, and $\mathrm{FD}(t=1.340$ and $p=$ $0.285)$ between groups. Patients with anti-NMDAR encephalitis demonstrated significantly lower scores in MoCA $(t=-3.350$ and $p=0.002)$, Auditory Verbal Learning Test-Immediate Recall (AVLT_IR) $(t=-4.099$ and $p<0.001)$, and Auditory Verbal Learning Test-Delayed Recall (AVLT_DR) $(t=-3.537$ and $p=0.001)$ compared to NCs (Table 1). All patients received first-line immunotherapy; three patients also received second-line immunotherapy, and three patients with ovarian teratomas underwent tumor resection (Supplemental Table S1).

\section{Gray matter atrophy}

Compared with NCs, patients with anti-NMDAR encephalitis showed significant gray matter atrophy in the left triangle part of the inferior frontal gyrus (trilFG.L), right triangle part of the inferior frontal gyrus (trilFG.R), and right precuneus (PCUN.R) (Figure 1 and Table 2). 
Table 2

Brain regions showing gray matter atrophy in patients with anti-N-methyl-D-aspartate receptor encephalitis

\begin{tabular}{|lllll|}
\hline Brain regions & Abbreviations & $\begin{array}{l}\text { Peak } \\
\text { intensity }\end{array}$ & $\begin{array}{l}\text { Cluster } \\
\text { size }\end{array}$ & $\begin{array}{l}\text { MNI } \\
\text { coordinates } \\
(\mathbf{x}, \mathbf{y}, \mathbf{z})\end{array}$ \\
\hline $\begin{array}{l}\text { Left triangle part of inferior frontal } \\
\text { gyrus }\end{array}$ & trilFG.L & -4.963 & 4838 & $42,33,6$ \\
\hline $\begin{array}{l}\text { Right triangle part of inferior frontal } \\
\text { gyrus }\end{array}$ & trilFG.R & -4.630 & 2730 & $-51,36,18$ \\
\hline Right precuneus & PCUN.R & -5.182 & 6730 & $18,-51,34$ \\
\hline MNI, Montreal Neurological Institute & & & & \\
\hline
\end{tabular}

\section{RSFC alterations}

The RSFC between the trilFG.L and left Heschl gyrus (HES.L)/right Heschl gyrus (HES.R), between the trilFG.R and HES.R, and between the PCUN.R and left cerebellum (CERE.L) were significantly lower in patients with anti-NMDAR encephalitis than in NC, whereas the opposite was true for the RSFC between the trilFG.R and left superior frontal gyrus (SFG.L) (Figure 2 and Table 3).

Table 3

Alterations of resting state functional connectivity in patients with anti-NMDAR encephalitis

\begin{tabular}{|llllll|}
\hline $\begin{array}{l}\text { Seed } \\
\text { regions }\end{array}$ & Brain regions & Abbreviations & $\begin{array}{l}\text { Peak } \\
\text { intensity }\end{array}$ & $\begin{array}{l}\text { Cluster } \\
\text { size }\end{array}$ & $\begin{array}{l}\text { MNI } \\
\text { coordinates } \\
\mathbf{( x , y , ~ z )}\end{array}$ \\
\hline trilFG.L & Right Heschl gyrus & HES.R & -5.141 & 203 & $51,-12,3$ \\
\hline & Left Heschl gyrus & HES.L & -5.216 & 107 & $-36,-30,12$ \\
\hline trilFG.R & Right Heschl gyrus & HES.R & -5.691 & 271 & $51,-9,3$ \\
\hline & $\begin{array}{l}\text { Left superior frontal } \\
\text { gyrus }\end{array}$ & SFG.L & 5.491 & 289 & $-21,54,36$ \\
\hline PCUN.R & Left cerebellum & CERE.L & -4.721 & 100 & $-3,-66,-30$ \\
\hline MNI, Montreal Neurological Institute & & & & \\
\hline
\end{tabular}

\section{Correlation results}

Partial correlation analyses controlled for age, sex, and education revealed that the gray matter volume in trilFG.R was positively correlated with AVLT_DR scores $(r=0.506$ and $p=0.027)$ and marginally correlated with AVLT_IR scores ( $r=0.452$ and $p=0.052$ ) in patients with anti-NMDAR encephalitis (Figure $3 A)$. Moreover, gray matter volume in trilFG.R was marginally correlated with disease duration in patients with anti-NMDAR encephalitis controlled for age, sex, and education (Figure 3B). The RSFC between 
trilFG.L and HES.R was positively correlated with AVLT_DR scores $(r=0.540$ and $p=0.021)$ and AVLT_IR scores $(r=0.558$ and $p=0.016)$ in patients with anti-NMDAR encephalitis controlled for age, sex, education, and FD (Figure $3 C$ ).

\section{Discussion}

Anti-NMDAR encephalitis is a type of severe autoimmune encephalitis. Clinical routine MRI findings are either normal or with mild changes in most patients with acute anti-NMDAR encephalitis (John et al., 2019). Moreover, $>75 \%$ of patients still suffer from prolonged cognitive deficits, even with timely access to adequate immunotherapy (McKeon et al., 2021; McKeon et al., 2018), indicating that these patients may have cerebral functional and/or structural changes. Indeed, most patients in this study showed normal routine clinical MRI results. However, we found structural and functional changes in some brain regions with high-resolution structural MRI and rs-fMRI.

Patients with anti-NMDAR encephalitis showed significant gray matter atrophy in bilateral trilFG and PCUN.R compared with NCs. Additionally, the RSFCs between trilFG.L and HES.L/HES.R, between trilFG.R and HES.R, and between PCUN.R and left CERE.L were significantly reduced in patients with anti-NMDAR encephalitis compared to NCs, yet the opposite was true for the RSFC between trilFG.R and SFG.L as assessed using seed-to-whole-brain voxel analyses. Additionally, decreased gray matter volume and RSFC were associated with memory deficits and disease duration. These findings suggest that these typical gray matter atrophies and altered RSFCs may represent key structural alterations related to the cognitive symptoms of anti-NMDAR encephalitis, and that combined multi-MRI and cognitive assessment are potentially valuable to assess prognosis and treatment efficacy.

Significant gray matter atrophy was observed in both trilFG.R and trilFG.L. These results were similar to the findings of our previous studies, which demonstrated decreased cerebral blood flow in both trilFG.R and trilFG.L in 15 patients with anti-NMDAR encephalitis compared with 15 NCs(Guo Y et al., 2020). The IFG is considered sensitive to auditory and phonological information and, consequently, related to verbal working memory (Zhu et al., 2020). Indeed, decreased gray matter volume in the IFG has been observed in patients with neurological diseases characterized by deficits in higher-order cognition, such as multiple sclerosis, Alzheimer's disease, and mild cognitive impairment (Rossi et al., 2016; Toko et al., 2021; Whitwell et al., 2008). Consistently with previous studies(Irish et al., 2014), we demonstrated that the decreased gray matter volume in trilFG.R was positively correlated with AVLT_DR scores and marginally correlated with AVLT_IR scores in patients with anti-NMDAR encephalitis, controlling for age, sex, and education. The AVLT score is used to assess verbal working memory (Xu et al., 2019) and was significantly decreased in the patients in this study compared with NCs. Moreover, we found that decreased gray matter volume in trilFG.R was marginally related to disease duration. Briefly, we presumed that the IFGs may be key contributors to anti-NMDAR encephalitis pathogenesis. Additionally, we identified significant decreases in gray matter volume in PCUN.R in patients with anti-NMDAR encephalitis compared with NCs, and this result is consistent with previous studies. Compared with NCs, patients with anti-NMDAR encephalitis were reported to have decreased white matter volume, amplitude 
of low frequency fluctuations (ALFF), and hypometabolism in PCUN (Cai et al., 2020; Liang et al., 2020; Wegner et al., 2014). Although no relationship between PCUN.R cortical atrophy and cognitive scores has been demonstrated, we cannot exclude their association since PCUN has been reported to be involved in the processing of working memory, and patients with cognitive deficits usually show hypoperfusion and disruptions of functional connectivity within PCUN (Ferri et al., 2016; Jia et al., 2018).

Brain structure atrophy may be accompanied by functional impairment. For example, altered gray matter volume and RSFC were observed in patients with neurological and psychiatric diseases, such as Parkinson's disease, major depression, and bipolar disorder, compared with NCs (Chen et al., 2018; Droby et al., 2021). Using the clusters derived from the VBM analysis of seed regions, we observed altered RSFCs between several regions in patients with anti-NMDAR encephalitis, indicating functional impairment in these brain areas. We primarily found decreased RSFC between trilFG.L and HES.L/HES.R and between trilFG.R and HES.R in patients with anti-NMDAR encephalitis compared with NCs. Previously, decreased HES RSFC has been observed in autoimmune diseases such as multiple sclerosis (Fu et al., 2019), and another report has demonstrated extensive damage to HES in patients with status epilepticus following viral meningoencephalitis (P.Pillion et al., 2014). Patients with seizures have been reported to be seizure-free following HES resection (Ferri et al., 2014). Additionally, an MRI study has found that patients with schizophrenia demonstrated significantly decreased RSFC between HES and IFG (Guo et al., 2014). Epileptic seizures and psychosis are two of the most typical clinical manifestations of antiNMDAR encephalitis (Dalmau et al., 2008).

We demonstrated a significant correlation between decreased RSFC and AVLT scores, which are used to assess verbal working memory (Xu et al., 2019). As we know, verbal working memory is divided into phonological store and articulatory rehearsal (Baddeley, 1992). The Heschl gyrus is a major component of the superior temporal gyrus and forms the primary auditory region (Fernandez et al., 2020); the IFG is also considered to play an important role in auditory and visual verbal information processing (Liu $\mathrm{X}$ et al., 2012; Zhu et al., 2020). Therefore, these two brain regions are both related to verbal working memory, thus supporting our observations.

Additionally, compared with NCs, patients with anti-NMDAR encephalitis had decreased RSFC between PCUN.R and left CERE.L. Previous studies have indicated that CERE showed high NMDAR expression besides the frontal lobe and hippocampus (Moscato et al., 2014; Skowronska et al., 2019; Wang and Xiao, 2020). Decreased ALFF were also observed in CERE and PCUN in patients with anti-NMDAR encephalitis (Cai et al., 2020), indicating functional impairment in these brain regions. Even though we did not find a direct link between the RSFC between these two regions and the memory performance, the disrupted connectivity between PCUN and CERE is an interesting finding for the pathological basis of anti-NMDAR encephalitis. Besides decreased RSFC in several brain regions, we also found increased RSFC between trilFG.R and SFG.L in patients with anti-NMDAR encephalitis compared with NCs. Moreover, a previous study has demonstrated a hyperintense lesion in SFG in a patient who tested positive for both anti-myelin oligodendrocyte glycoprotein and anti-NMDAR antibodies (Nagata et al., 2018), supporting the results of this study. Increased RSFC is considered a compensation after acute 
inflammatory injury or a result of the proliferation of glial cells during recovery (Cai et al., 2020). As the neural activity requires cerebral blood perfusion to supply oxygen and nutrients, elevated perfusion tends to induce increased neural activity (Poornima et al., 2016). Moreover, increased cerebral blood perfusion was also observed in patients with anti-NMDAR encephalitis due to the increased permeability of the blood-brain barrier (Guo Y et al., 2020; Suárez et al., 2016).

This study has several limitations. First, its small sample size limited statistical efficiency, and more patients are still needed to verify our results. Second, abnormal MRI findings with different lesions were identified in nearly $50 \%$ of patients at onset, and their potential effects are concerning. Third, the the results of this study were limited by its cross-sectional design; longitudinal studies further exploring the neural mechanism of anti-NMDAR encephalitis are warranted.

\section{Conclusion}

In conclusion, our study found gray matter atrophy and altered RSFC in the bilateral trilFG and right PCUN in patients with anti-NMDAR encephalitis. The combination of structural and functional MRI demonstrates a characteristic pattern of pathological alterations in the aforementioned patients. We believe that assessing these characteristic brain alterations may provide a theoretical basis for exploring promising therapeutic strategies for anti-NMDAR encephalitis.

\section{Declarations}

\section{Acknowledgements}

No.

\section{Compliance with Ethical Standards}

Ethical Approval All the experiment procedures performed in this study was approved by the ethics committee of the Anhui Medical University and was accordance with the Helsinki declaration and its later amendments or comparable ethical standards. All participants provided informed written consent.

Consent to Participate The written informed consent for publication was obtained from all participants included in the study.

Consent to Publish All the authors have read and approved the submission.

Author Contributions Yuanyuan Guo, Jinping Xu, and Yanghua Tian designed the study. Yuanyuan Guo, Xinyi Lv, Juanjuan Zhang, and Chenglong Li collected the data. Jinping $X u$ analyzed the data. Yuanyuan Guo drafted the manuscript. Juanjuan Zhang, Ling Wei, Nong Zhou, Jinping Xu, Yanghua Tian, and Kai Wang revised the draft. 
Funding This work was supported by funding from the National Natural Science Foundation of China (32071054), the National Natural Science Foundation of China (62006220), and the Shenzhen Science and Technology Research Program (JCYJ20200109114816594).

Competing Interests The authors declare that they have no competing interests.

Data Availability All data generated or used during the study are available from the corresponding author by request.

\section{References}

1. Bacchi, S., Franke, K., Wewegama, D., Needham, E., Patel, S., \& Menon, D. (2018). Magnetic resonance imaging and positron emission tomography in anti-NMDA receptor encephalitis: A systematic review. J Clin Neurosci, 52, 54-59. doi:10.1016/j.jocn.2018.03.026

2. Bach, L. J. (2014). Long term rehabilitation management and outcome of anti-NMDA receptor encephalitis: case reports. NeuroRehabilitation 35(4), 863-875. doi:10.3233/NRE-141176

3. Baddeley, A. (1992). Working memory. Science, 255(5044), 556-559. doi:10.1126/science.1736359

4. Brier, M. R., Day, G. S., Snyder, A. Z., Tanenbaum, A. B., \& Ances, B. M. (2016). N-methyl-D-aspartate receptor encephalitis mediates loss of intrinsic activity measured by functional MRI. J Neurol, 263(6), 1083-1091. doi:10.1007/s00415-016-8083-6

5. Cai, L., Liang, Y., Huang, H., Zhou, X., \& Zheng, J. (2020). Cerebral functional activity and connectivity changes in anti-N-methyl-D-aspartate receptor encephalitis: A resting-state fMRI study. Neuroimage Clin, 25102189, doi:10.1016/j.nicl.2020.102189

6. Carsten, F., Ute, A., Kopp, A., Pajkert, Janina, R., \& Behrens (2016). Structural Hippocampal Damage Following Anti-N-Methyl-D-Aspartate Receptor Encephalitis. Biological Psychiatry, 79(9), 727-734. doi:10.1016/j.biopsych.2015.02.024

7. Chen, L., Wang, Y., Niu, C., Zhong, S., Hu, H., Chen, P. ... Huang, R. (2018). Common and distinct abnormal frontal-limbic system structural and functional patterns in patients with major depression and bipolar disorder. Neuroimage Clin 2042-50. doi:10.1016/j.nicl.2018.07.002

8. Dalmau, J. (2016). NMDA receptor encephalitis and other antibody-mediated disorders of the synapse: The 2016 Cotzias Lecture. Neurology 87(23), 2471-2482.

doi:10.1212/WNL.0000000000003414

9. Dalmau, J., Gleichman, A. J., Hughes, E. G., Rossi, J. E., Peng, X., Lai, M. ... Lynch, D. R. (2008). AntiNMDA-receptor encephalitis: case series and analysis of the effects of antibodies. Lancet Neurol, 7(12), 1091-1098. doi:10.1016/S1474-4422(08)70224-2

10. Dalmau, J., Lancaster, E., Martinez-Hernandez, E., Rosenfeld, M. R., \& Balice-Gordon, R. (2011). Clinical experience and laboratory investigations in patients with anti-NMDAR encephalitis. Lancet Neurol, 10(1), 63-74. doi:10.1016/S1474-4422(10)70253-2 
11. Droby, A., Pelosin, E., Putzolu, M., Bommarito, G., Marchese, R., Mazzella, L. ... Inglese, M. (2021). A Multimodal Imaging Approach Demonstrates Reduced Midbrain Functional Network Connectivity Is Associated With Freezing of Gait in Parkinson's Disease. Front Neurol 12583593-583593. doi:10.3389/fneur.2021.583593

12. Fernandez, L., Velasquez, C., Garcia Porrero, J. A., de Lucas, E. M., \& Martino, J. (2020). Heschl's gyrus fiber intersection area: a new insight on the connectivity of the auditory-language hub. Neurosurg Focus, 48(2), E7. doi:10.3171/2019.11.FOCUS19778

13. Ferri, J., Schmidt, J., Hajcak, G., \& Canli, T. (2016). Emotion regulation and amygdala-precuneus connectivity: Focusing on attentional deployment. Cogn Affect Behav Neurosci, 16(6), 991-1002. doi:10.3758/s13415-016-0447-y

14. Ferri, L., Bisulli, F., Nobili, L., Tassi, L., Licchetta, L., Mostacci, B. ... Tinuper, P. (2014). Auditory aura in nocturnal frontal lobe epilepsy: a red flag to suspect an extra-frontal epileptogenic zone. Sleep Med, 15(11), 1417-1423. doi:10.1016/j.sleep.2014.06.019

15. Finke, C., Kopp, U. A., Scheel, M., Pech, L. M., Soemmer, C., Schlichting, J. ... Paul, F. (2013). Functional and structural brain changes in anti-N-methyl-D-aspartate receptor encephalitis. Ann Neurol, 74(2), 284-296. doi:10.1002/ana.23932

16. Fox, M. D., \& Raichle, M. E. (2007). Spontaneous fluctuations in brain activity observed with functional magnetic resonance imaging. Nat Rev Neurosci, 8(9), 700-711. doi:10.1038/nrn2201

17. Fu, J., Chen, X., Gu, Y., Xie, M., Zheng, Q., Wang, J. ... Li, Y. (2019). Functional connectivity impairment of postcentral gyrus in relapsing-remitting multiple sclerosis with somatosensory disorder. Eur J Radiol, 118, 200-206. doi:10.1016/j.ejrad.2019.07.029

18. Guo, Q., Tang, Y., Hui, L., Zhang, T., \& Wang, J. (2014). Both volumetry and functional connectivity of Heschl's gyrus are associated with auditory P300 in first episode schizophrenia. Schizophrenia Research, 160(1-3), 57-66. doi:10.1016/j.schres.2014.10.006

19. Guo, Y., Lv, X., Wu, Y., Chen, Y., Wei, Q., Zhou, N., \& Wang, K., Y., T (2020). Cognitive function and cerebral blood perfusion changes in patients with anti-N-methyl-d-aspartate receptor encephalitis. Zhonghua Yi Xue Za Zhi, 100(25), 1942-1946. doi:10.3760/cma.j.cn112137-20200108-00050

20. Irani, S. R., Katarzyna, B., Patrick, W., Luigi, Z., Susan, M., Zandi, M. S. ... David, B. (2010). N-methyl-Daspartate antibody encephalitis: temporal progression of clinical and paraclinical observations in a predominantly non-paraneoplastic disorder of both sexes. Brain A Journal of Neurology, (6), 16551667. doi:10.1093/brain/awq113

21. Irish, M., Piguet, O., \& Hornberger, M. (2014). Common and Unique Gray Matter Correlates of Episodic Memory Dysfunction in Frontotemporal Dementia and Alzheimer's Disease. Human brain mapping, 35(4), 1422-1435. doi:10.1002/hbm.22263

22. Jia, X., Li, Y., Li, K., Liang, P., \& Fu, X. (2018). Precuneus Dysfunction in Parkinson's Disease With Mild Cognitive Impairment. Front Aging Neurosci, 10427, doi:10.3389/fnagi.2018.00427

23. Xu, J., Guo, Y., Li, J., Lv, X., Zhang, J., Zhang, J. ... Tian, Y. (2021). Progressive cortical and sub-cortical alterations in patients with anti-N-methyl-D-aspartate receptor encephalitis. Journal of 
neurologypublished online ahead of print. doi:10.1007/s00415-021-10643-1

24. John, C. M., Mathew, D. E., Abdelaziz, M., Mahmoud, A. A. H., AlOtaibi, A. D., \& Sohal, A. P. S. (2019). Anti-N-methyl-d-aspartate Receptor Encephalitis: A Case Series and Review of the Literature. $J$ Pediatr Neurosci, 14(4), 180-185. doi:10.4103/jpn.JPN_83_19

25. Lau, C. G., \& Zukin, R. S. (2007). NMDA receptor trafficking in synaptic plasticity and neuropsychiatric disorders. Nat Rev Neurosci, 8(6), 413-426. doi:10.1038/nrn2153

26. Liang, Y., Cai, L., Zhou, X., Huang, H., \& Zheng, J. (2020). Voxel-based analysis and multivariate pattern analysis of diffusion tensor imaging study in anti-NMDA receptor encephalitis.

Neuroradiology, 62(2), 231-239. doi:10.1007/s00234-019-02321-x

27. Liu, X., Lauer, K. K., Ward, B. D., Rao, S. M., Li, S. J., \& AG, H. (2012). Propofol disrupts functional interactions between sensory and high-order processing of auditory verbal memory. Human Brain Mapping, 33(10), 2487-2498. doi:10.1002/hbm.21385

28. McKeon, G., Parker, S., Warren, N., \& Scott, J. G. (2021). The Patient Experience of Recovery Following Anti-NMDA Receptor Encephalitis: A Qualitative Content Analysis. J Neuropsychiatry Clin Neurosci, 33(1), 57-63. doi:10.1176/appi.neuropsych.20030049

29. McKeon, G. L., Robinson, G. A., Ryan, A. E., Blum, S., Gillis, D., Finke, C., \& Scott, J. G. (2018). Cognitive outcomes following anti-N-methyl-D-aspartate receptor encephalitis: A systematic review. J Clin Exp Neuropsychol, 40(3), 234-252. doi:10.1080/13803395.2017.1329408

30. Moscato, E. H., Peng, X., Jain, A., Parsons, T. D., Dalmau, J., \& Balice-Gordon, R. J. (2014). Acute mechanisms underlying antibody effects in anti-N-methyl-D-aspartate receptor encephalitis. Ann Neurol, 76(1), 108-119. doi:10.1002/ana.24195

31. Nagata, S., Nishimura, Y., \& Mitsuo, K. (2018). [A case of anti-myelin oligodendrocyte glycoprotein (MOG) and anti-N-methyl-D-aspartate (NMDA) receptor antibody-positive encephalitis with optic neuritis]. Rinsho Shinkeigaku, 58(10), 636-641. doi:10.5692/clinicalneurol.cn-001194

32. Shiffler, P. P. J. E., Hoon, D. H., \& .Lin, A. D. M. (2014). D., Severe auditory processing disorder secondary to viral meningoencephalitis. International Journal of Audiology 53427-431. doi:10.3109/14992027.2014.892644

33. Peer, M., Pruss, H., Ben-Dayan, I., Paul, F., Arzy, S., \& Finke, C. (2017). Functional connectivity of largescale brain networks in patients with anti-NMDA receptor encephalitis: an observational study. Lancet Psychiatry, 4(10), 768-774. doi:10.1016/S2215-0366(17)30330-9

34. Poornima, V., Chopp, M., Jieli, \& Chen (2016). New insights into coupling and uncoupling of cerebral blood flow and metabolism in the brain. Croatian Medical Journal, 57(3), 223-228. doi:10.3325/cmj.2016.57.223

35. Power, J. D., Barnes, K. A., Snyder, A. Z., Schlaggar, B. L., \& Petersen, S. E. (2012). Spurious but systematic correlations in functional connectivity MRI networks arise from subject motion. Neuroimage, 59(3), 2142-2154. doi:10.1016/j.neuroimage.2011.10.018

36. Rossi, R., Pievani, M., Jarvenpaa, T., Testa, C., Koskenvuo, M., Raiha, I. ... Laakso, M. P. (2016). Voxelbased morphometry study on monozygotic twins discordant for Alzheimer's disease. Acta 
Neurologica Scandinavica, 133(6), 427-433. doi:10.1111/ane.12480

37. Satterthwaite, T. D., Elliott, M. A., Gerraty, R. T., Ruparel, K., Loughead, J., Calkins, M. E. ... Wolf, D. H. (2013). An improved framework for confound regression and filtering for control of motion artifact in the preprocessing of resting-state functional connectivity data. Neuroimage 64240-256. doi:10.1016/j.neuroimage.2012.08.052

38. Skowronska, K., Obara-Michlewska, M., Zielinska, M., \& Albrecht, J. (2019). NMDA Receptors in Astrocytes: In Search for Roles in Neurotransmission and Astrocytic Homeostasis. Int J Mol Sci, 20(2), 309. doi:10.3390/ijms20020309

39. Suárez, J., Domínguez, M., Gómez, M., Portilla, J. C., Gómez, M., \& Casado, I. (2016). Brain perfusion SPECT with (99m)Tc-HMPAO in the diagnosis and follow-up of patients with anti-NMDA receptor encephalitis. Neurologia 33(9), 622-623. doi:10.1016/j.nrl.2016.05.019

40. Toko, M., Kitamura, J., Ueno, H., Ohshita, T., Nemoto, K., Ochi, K. ... Maruyama, H. (2021). Prospective Memory Deficits in Multiple Sclerosis: Voxel-based Morphometry and Double Inversion Recovery Analysis. Intern Med, 60(1), 39-46. doi:10.2169/internalmedicine.5058-20

41. Viaccoz, A., Desestret, V., Ducray, F., Picard, G., Cavillon, G., Rogemond, V. ... Delattre, J. Y., J, H (2014). Clinical specificities of adult male patients with NMDA receptor antibodies encephalitis. Neurology, 82(7), 556-563. doi:10.1212/WNL.0000000000000126

42. Wang, H., \& Xiao, Z. (2020). Current Progress on Assessing the Prognosis for Anti-N-Methyl-DAspartate Receptor (NMDAR) Encephalitis. Biomed Res Int, 20207506590, doi:10.1155/2020/7506590

43. Wegner, F., Wilke, F., Raab, P., Tayeb, S. B. ... Boeck, A. (2014). Anti-leucine rich glioma inactivated 1 protein and anti-N-methyl-D-aspartate receptor encephalitis show distinct patterns of brain glucose metabolism in 18F-fluoro-2-deoxy-d-glucose positron emission tomography. Bmc Neurology, 14(1), 136. doi:10.1186/1471-2377-14-136

44. Whitwell, J. L., Shiung, M. M., Przybelski, S. A., Weigand, S. D., Knopman, D. S., Boeve, B. F. ... Jack, C. R. (2008). MRI patterns of atrophy associated with progression to AD in amnestic mild cognitive impairment. Neurology, 70(7), 512-520. doi:10.1212/01.wnl.0000280575.77437.a2

45. Xu, J., Guo, Y., Li, J., Lv, X., Zhang, J., Zhang, J. ... Tian, Y. (2021). Progressive cortical and sub-cortical alterations in patients with anti-N-methyl-D-aspartate receptor encephalitis. Journal of Neurology, (4), doi:10.1007/s00415-021-10643-1

46. Xu, J., Wang, J., Bai, T., Zhang, X., Li, T., Hu, Q.... Wang, K. (2019). Electroconvulsive Therapy Induces Cortical Morphological Alterations in Major Depressive Disorder Revealed with Surface-Based Morphometry Analysis. Int J Neural Syst, 29(7), 1950005. doi:10.1142/S0129065719500059

47. Zhu, R., Luo, Y., Wang, Z., \& You, X. (2020). Modality effects in verbal working memory updating: Transcranial direct current stimulation over human inferior frontal gyrus and posterior parietal cortex. Brain Cogn, 145105630, doi:10.1016/j.bandc.2020.105630

\section{Figures}




\section{Figure 1}

Differences in gray matter volume between patients with anti-N-methyl-D-aspartate receptor (NMDAR) encephalitis and normal controls (NCs). (A) Brain regions showing gray matter atrophy were compared between groups using a two-sample t-test, with sex, age, and education as covariates. Results were corrected using a Gaussian random field (GRF) with a voxel level of $p<0.001$ and cluster level of $p<$ 0.05. (B) Compared with NCs (in green), the mean regional gray matter volume was significantly reduced in patients with anti-NMDAR encephalitis (in red). A general linear model (GLM) was performed with sex, age, education, and frame-wise displacement as covariates. Stars represent significant results with $p$ value $<0.05$. Abbreviations for brain regions are listed in Table 2 .

\section{Figure 2}

Comparison of resting state functional connectivity (RSFC) in patients with anti-N-methyl-D-aspartate receptor (NMDAR) encephalitis and normal controls (NCs). The first column is for seed regions, the middle column is for RSFC patterns, and the last is for mean RSFC. Group differences were analyzed using a two-sample t-test, with sex, age, education, and frame-wise displacement used as covariates. The results were corrected using a Gaussian random field with a voxel level of $p<0.001$ and cluster level of $p$ $<0.05$. Mean RSFCs were compared using a GLM, with sex, age, education, and frame-wise displacement as covariates. Stars represent significant results with $p<0.05$. Abbreviations for brain regions are listed in Table 3.

\section{Figure 3}

Correlation results. (A) The gray matter volume in the right triangle part of the inferior frontal gyrus (trilFG.R) was positively correlated with AVLT_DR scores and marginally correlated with AVLT_IR scores in patients with anti-N-methyl-D-aspartate receptor encephalitis controlled for age, sex, and education. (B) The resting state functional connectivity (RSFC) between the left triangle part of the inferior frontal gyrus and right Heschl gyrus (HES.R) was positively correlated with AVLT_DR scores and marginally correlated with AVLT_IR scores in patients with anti-N-methyl-D-aspartate receptor encephalitis controlled for age, sex, education, and frame displacement. (C) The RSFC between trilFG.R and HES.R was marginally correlated with disease course in patients with anti-N-methyl-D-aspartate receptor encephalitis.

\section{Supplementary Files}

This is a list of supplementary files associated with this preprint. Click to download. 
- AuthorChecklist.pdf

- Supplementarymaterial.docx 